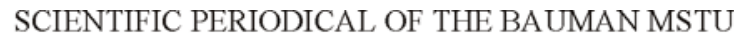 \\ SCIENCE and EDUCATION
}

EL No FS77 - 48211. No0421200025. ISSN 1994-0408

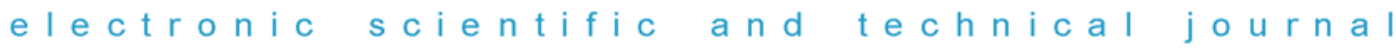

\section{Synthesis of orientation mechanisms for the space observatory «Millimetron». 1. Capabilities of parallel mechanisms for orientation of the space observatory «Millimetron»}

\# 01, January 2013

DOI: 10.7463/0113.0534292

Artemenko Yu.N.

The Lebedev Physical Institute of the Russian Academy of Sciences. Astro Space Center altishenko@yahoo.com

The present paper opens a series of 4 publications dedicated to the synthesis of orientation mechanisms of "Millimetron" space telescope being itself the introductory one. The article series is published under general edition by Yu.N. Artemenko and A.P. Karpenko.

The "Millimetron" project is currently being developed under scientific guidance of the Astrocosmic Center (ACC) of the Physical Institute named after P.N. Lebedev of RAN named after Academician N.S. Kardashev, of scientists and specialists of ACC AIAN, NPO named after S.A. Lavochkin and OAO "Satellite Information Systems” named after M.F. Reshetnev of Roskosmos in cooperation with a number of national and international organizations in accordance with the Federal Space Program. The objective of the project is the construction of a space observatory with a $10 \mathrm{~m}$ cryogenic radio-telescope for the wave band $20 \mathrm{~mm} . . .20 \mu \mathrm{m}$. The purpose of "Millimetron" observatory will be the study of different types of objects in the Universe with ultrahigh sensitivity (as single probe) and ultrahigh angular resolution (as multi-station terrestrial-orbital interferometry system) in IR and millimeter wave band [1]. The ultrahigh sensitivity of the space telescope is achieved due to cryogenic cooling with liquid helium of the antenna reflector, heat protection screens, and the reception hardware (Fig. 1.1). The sensitivity of such telescope will be equal to the sensitivity of a 100 $\mathrm{m}$ space telescope without cooling. 


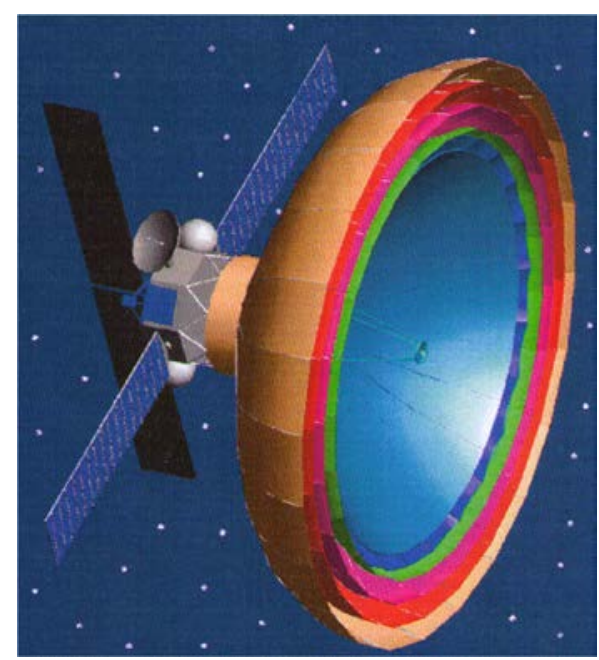

Fig. 1.1 - General view of one of the versions of "Millimetron” space observatory

The observatory will be mounted on the space platform of "Navigator" type used for "Radioastron" project and comprise an automatically unfolded triplereflector antenna with a diameter of $10 \mathrm{~m}$ with coaxial active and passive heat protection screens. The main reflector of the antenna, the adjacent heat protection screen, as well as the cryogenic container of the scientific hardware are cooled with liquid helium down to $4.5 \mathrm{~K}$, and the reception hardware - down to $0.2 \mathrm{~K}$. The main reflector is constituted by unfolded and fastened together rigid precision blades with adaptable reflection surface. The counterreflector of the antenna with $57 \mathrm{~cm}$ diameter is located within the main reflector in the primary focus of the antenna preventing influence thereon of the thermal radiation of the Sun and the Earth and the Zodiac light. The counterreflector is equipped with a hexapod mechanism for the adjustment of the antenna after unfolding. The third flat rotary reflector is located in the area of the secondary focus if the antenna in the cryogenic container and has a 2D drive for the alignment of the received radiation axis with one of the receivers located around this reflector and for fine tuning.

The frequency of the own vibration of the antenna structure is approximately $5 \mathrm{~Hz}$, and that of the heat-protection screens is approximately $3 \mathrm{~Hz}$. The accuracy of the active surface of the main reflector of the antenna (rms deviation) during the telescope operation should not exceed $10 \mu \mathrm{m}$. The aiming accuracy of the telescope on the studies objects should be max. $1^{\prime \prime}$, and the stabilization accuracy should be max. $0,2^{\prime \prime}$. 
The ultrahigh resolution of the telescope us achieved as a result of its joint operation with big terrestrial telescopes in interferometry mode at a distance of 1.5 million $\mathrm{km}$ from the Earth. For gravity impact compensation of the Sun and the Earth by centrifugal force the telescope is planned to be launched to the orbit in the area of the antihelical Lagrange point $L 2$ with the orbit perpendicular to the ecliptic plane.

During the development of "Millimetron" observatory several design solutions of the telescope were considered which ensure the preset technical characteristics. In the aspect of the orientation system of the telescope for aiming on object sources two development ways can be identified.

First way is basing on a constant solar alignment of the longitudinal symmetry axis of the observatory and its radiation screens for its protection against the radiation of the Sun and the Earth (Fig. 1.2).
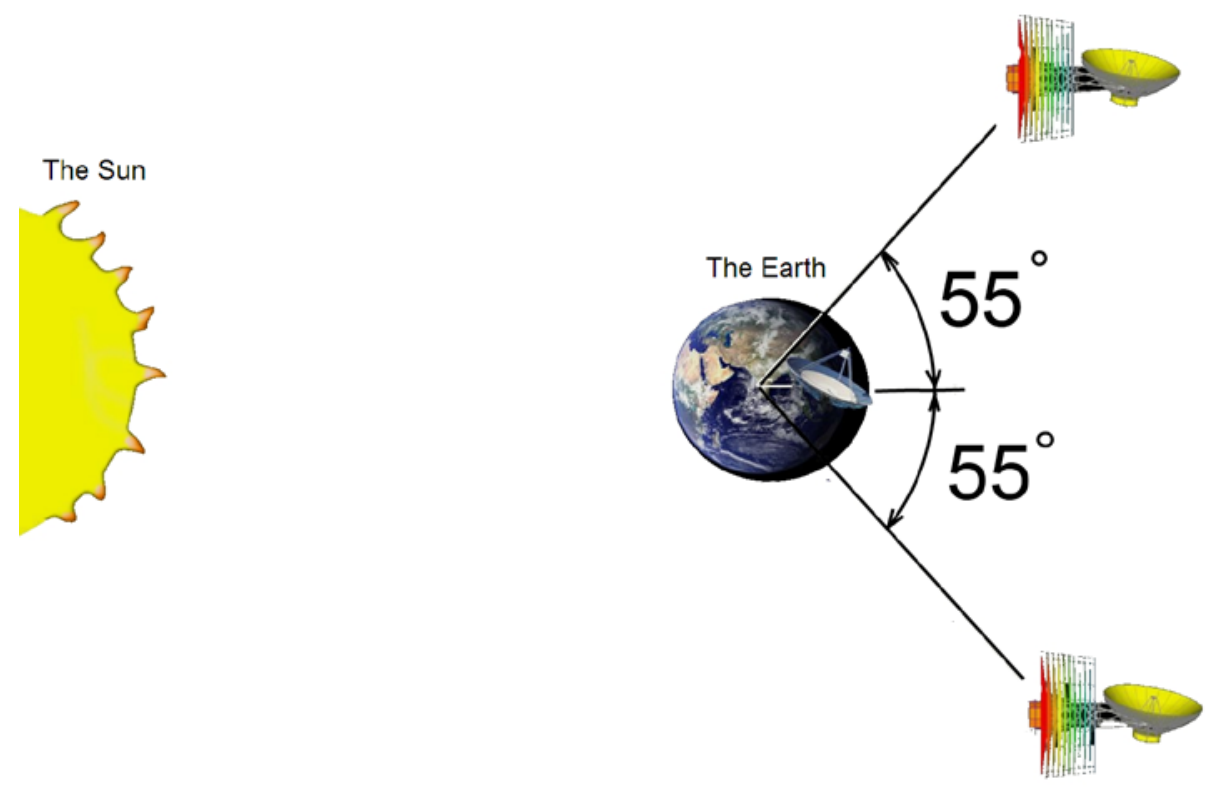

Fig. 1.2. Solar alignment of "Millimetron”

For the alignment of the telescope in this case a rotation of the antenna is required in relation to the auxiliary module with jet engines of correction, orientation and stabilization, as well as flywheel motors. Thereby the antenna itself should be displaced from the nearest heat protection screen by a distance exceeding the radius of the antenna in order not to collide with the antenna at a $90^{\circ}$ swing. One of such design versions of the observatory using a transformable frame as antenna support is presented in Fig. 1.3a. Thereby the observatory takes a 
protruded dumbbell shape on one side whereof the telescope is mounted, and on the other side the auxiliary module is placed. The gravity center of the observatory finds itself approximately in the middle of the dumbbell whereas the weights of the telescope and the auxiliary module are almost equal. The location of the gravity center of the observatory is in this case highly dependent on the gravity center of the telescope during its re-aiming operations causing the necessity for a compensation of arising torques with the engines of the auxiliary module.

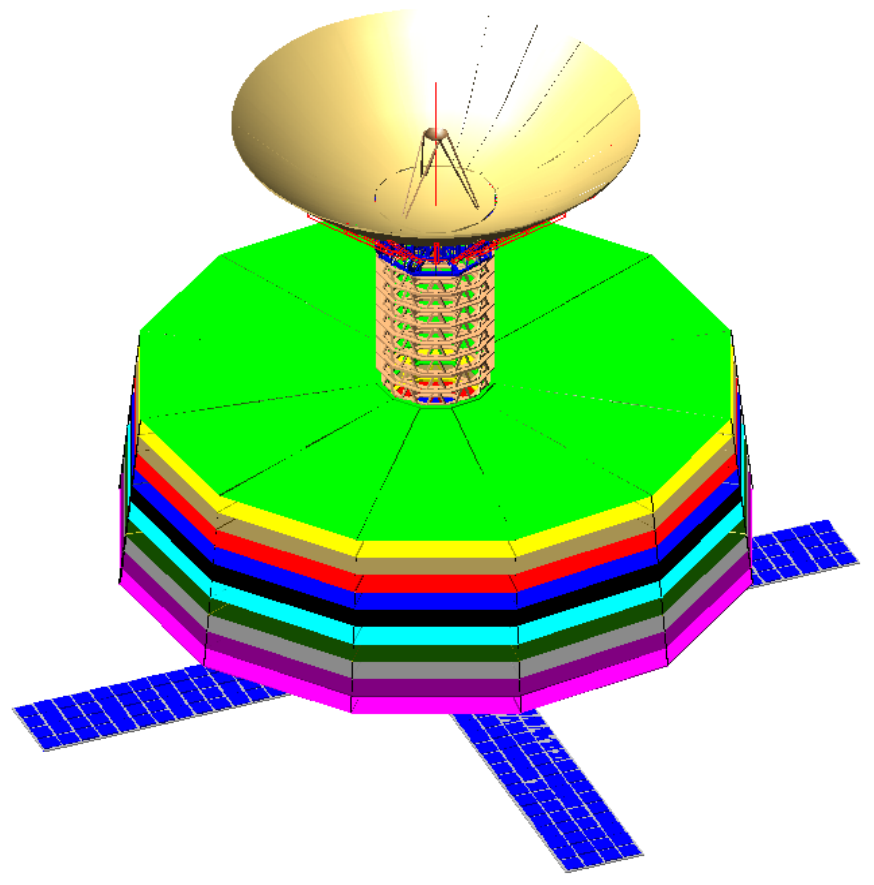

a) 


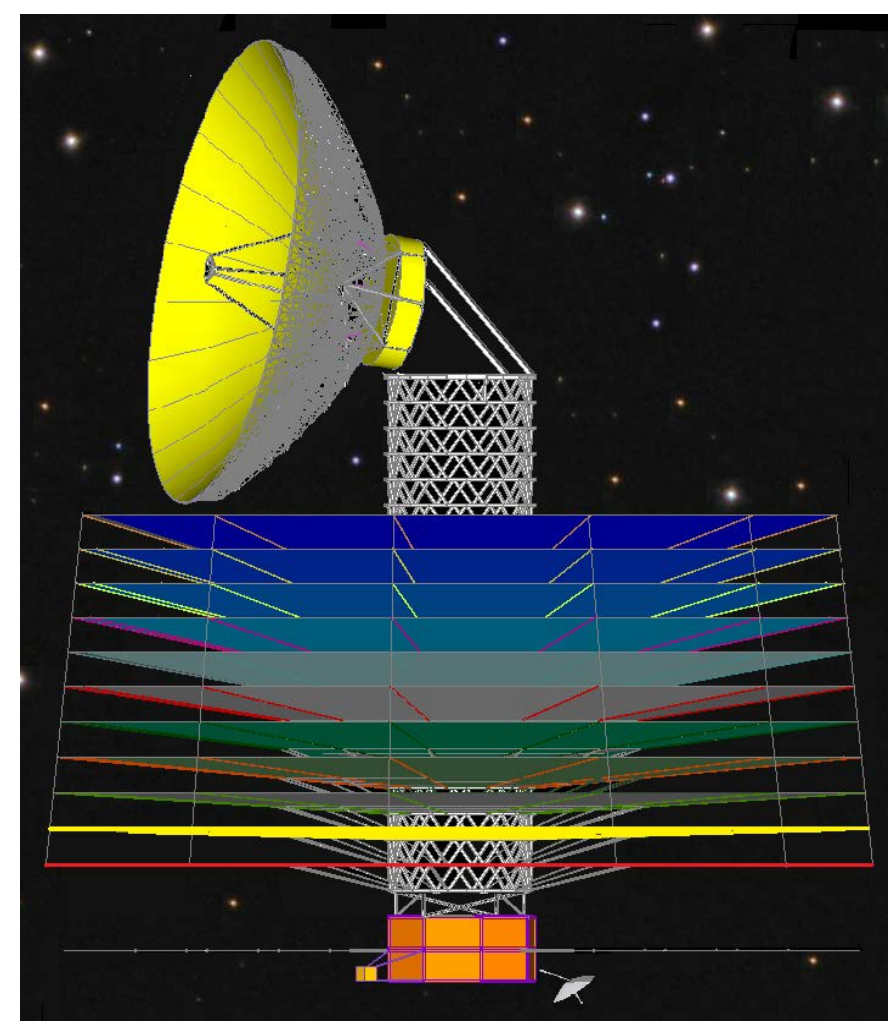

b)

Fig. 1. 3 Observatory design versions

The simplest mechanism of one-plane swing of the telescope proceeds from the assumption that the cryogenic container will be fastened to the edge of the support frame (Fig. 1.3b). However, the use of such mechanism will cause the gravity center of the telescope displace from the longitudinal symmetry axis by up to $1,5 \mathrm{~m}$ whereas the displacement if the gravity center of the observatory as a total will be up to $1 \mathrm{~m}$. A compensation of the torques arising thereby with the flywheel motors appears impossible, whereas the use of the jet engines of the auxiliary module is restricted by the fuel reserve which needs to be extended over the required life period of 10 years.

Thus, the indispensable requirement for the telescope alignment by means of its rotation is the preservation of the gravity center of the telescope on the longitudinal axis of the observatory or very close thereto. Along with the minimizing of the arising torques this requirement is stipulated by the fact that after the aiming of the telescope on the object its stabilization is required for the receipt of the radiation. Dependent on the type of the object and the scientific research program the required stabilizing time of the telescope will vary from 15 minutes to several hours. For telescope stabilizing the torques stipulated by external factors like light pressure and the solar wind (flux of solar plasma caused 
by Sun bursts). It can be achieved by alignment on the longitudinal axis of the observatory its gravity center and the geometrical center of the external screen. To meet the set forth requirements the swinging mechanism of the telescope should, along with the preservation of the set position of the gravity center, provide the opportunity of correction of this position. The swing mechanisms of the observatory handled in the second and the third publications of the series ensure conformity with these requirements.

The second article of the series is dedicated to an alternative design of the swing drive of the telescope based on a multisectional manipulator of parallel structure. The paper presents the software developed by the authors which performs the automatic generation of $3 D$ models of the manipulator by means of Solid Works and "heavy" graphic system NX 7.5 and the application "Simulation of kinematic mechanisms". By means of those models several manipulators have been studied. Ibidem the description of the developed application for forward and reversed position problem for the multisectional manipulator. Some results of studies performed in that application are presented. Beside that the same article handles the Simulink model of the controlled manipulators. A report on the study of kinematics and dynamics of transient states of the manipulator is provided.

The suggested multisectional manipulator for the aiming of the cosmic telescope "Millimetron" will perform the following functions: unfolding of the telescope from transportation into operation position; angular aiming of the telescope within the segment limited by the cooling system screens; maintaining a constant position of the gravity center of the telescope and the observatory (or with very small displacement) during aiming operations; positional correction of the gravity centers of the telescope and the observatory; alignment of the observatory; lowering of stabilizing vibrations of the telescope; unloading of the flywheel motors and other functions.

The third article considers the design variant of the slewing drive of the telescope based on double pendulum and double yoke principle. A design generation method of flat six-link mechanisms with three degrees of freedom ensuring such alteration of the antenna orientation of the cosmic telescope that leaves the center of gravity of this antenna intact. The method allows determining all unknown dimensions of the links of the mechanism, their swing angles as well as the power transmission function of the mechanism. 
The mechanisms of parallel structure handled in the second and the third articles could not, in spite of all their attractiveness and matching all the requirements to the aiming system of "Millimetron", be accepted for implementation since the design solutions of the telescope developed on their basis could not ensure the performance of the mission of the probe - the implementation of a cryogenic telescope. The handled mechanisms should reliably work at extra low temperatures $(4.5 \mathrm{~K})$ with all the numerous joints, motors and sensors; they cause considerable bending of power and data cables, as well as of the liquid helium hoses connecting the cryogenic container with the auxiliary module. Such mechanisms also scarcely can be included by the confined space under the shell of the carrier rocket. Nevertheless the performed design work of the parallel structure mechanism can be helpful in other spacecraft projects.

The second way of the design solution of the telescope in the aspect of the aiming control under the consideration of the cryogenic design is based on traditional designs of terrestrial telescopes. The antenna is mounted on the space platform via bottom connection frame, the "warm" hardware container and the top connection frame (Fig. 1.1). The aiming of the telescope is performed by flywheel motors installed in the auxiliary module by means of the orientation of the whole observatory. Thereby for protection of the telescope against the thermal radiation of the Sun and the Earth by means of the screens the swing angles of the telescope will not exceed $30^{\circ}$ from the Sun direction. This circumstance will reduce the number of would-be observation objects. Still, this number remains sufficient for carrying out of the scientific research program of the project. The aiming of the telescope by means of the turn of the observatory leads to a non-uniform irradiation of the Sun onto the external screen as to its geometric and gravity center which is accompanied by its involution and the necessity for the stabilization system to operate constantly. This will, in its turn, cause vibrations of the observatory structure. Thus, this design variant of the structural design of the telescope requires a system of active vibration damping and high-precision aiming based on parallel structure mechanisms.

The main contents of the fourth article of the series is the architectural concept of an intellectual system of vibration damping and high-precision aiming of the telescope of "Millimetron". The requirement for such system for any observatory design is arising from the requirement for its high precision. It should 
be stressed that orientation and stabilizing systems of existing and promising spacecrafts are not capable of ensuring the required aiming quality by traditional means. Beside that big space antennae are prolonged structures of usually low rigidity unfolding in the orbit. That is why the low-frequency vibrations of the structure of the antenna of a spacecraft caused by micro dynamic impacts during the orbital flight can cause a deviation of the active surface of the reflector axis beyond the tolerances, and as a sequence the radio technical characteristics of the antenna will get worse. Data are available that high-frequency vibrations of the structural elements do not fade under cryogenic conditions, once occurred. Thus, the problem of vibrations compensation, high-precision aiming and stabilizing is vital for the construction of the space telescope "Millimetron".

The progress of the work on the generation of the design of the orientation mechanisms of the cosmic telescope "Millimetron" requires the following problems be solved:

- Calculation of the structural rigidity;

- Definition of proprietary vibrations frequency of the structure;

- calculation of inertia torques to be compensated by the flywheel motors of the spacecraft during the antenna orientation;

- generation of rational trajectories of the antenna during the positioning;

- generation of classical adaptive and field bus control of the antenna drives.

The series of papers applies a two-level enumeration of text fragments, formulas, figures and tables. The first digit of the number is the publication number and the second digit is the number of the relevant object.

\section{References}

1. Wild W., Kardashev N.S., Babakin N.G., Arkhipov V.Y., Vinogradov I.S., Andreyanov V.V., Fedorchuk S.D., Myshonkova N.V., Alexsandrov Y.A., Novokov I.D., Goltsman G.N., Cherepaschuk A.M., Shustov B.M., Vystavkin A.N., Koshelets V.P., Vdovin V.F., de Graauw Th., Helmich F., Vd Tak F., Shipman R., Baryshev A., Gao J.R., Khosropanah P., Roelfsema P.. Barthel P., Spaans M., Mendez M., Klapwijk T., Israel F., Hogerheijde M., Vd Werf P., Cernicharo J., Martin-Pintado J., Planesas P., Gallego J.D., Beaudin G., Krieg J.M., Gerin M., Pagani L., Saraceno P., di Giorgio A.M., Cerulli R., Orfei R., Spinoglio L., Piazzo L., Liseau R., Belitsky V., Cherednichenko S., Poglitsch A., Raab W., Guesten R., Klein B., Stutzki J., Honingh N., Benz A., Murphy A., Trappe N., Räisänen A. Millimetron - a large Russian-European submillimeter space observatory. Experimental Astronomy, 2009, vol. 23, no. 1, pp. 221-244. DOI: 10.1007/s10686-0089097-6 